\title{
Wnt Signaling Specifies Anteroposterior Progenitor Zone Identity in the Drosophila Visual Center
}

\author{
Takumi Suzuki, ${ }^{1 \star}$ Olena Trush, ${ }^{2 \star}$ Tetsuo Yasugi, ${ }^{3}$ Rie Takayama, ${ }^{3,4}$ and Makoto Sato ${ }^{1,2,3,4}$ \\ ${ }^{1}$ Laboratory of Developmental Neurobiology, Brain/Liver Interface Medicine Research Center, ${ }^{2}$ Graduate School of Medical Sciences, and ${ }^{3}$ Mathematical \\ Neuroscience Unit, Institute for Frontier Science Initiative, Kanazawa University, Ishikawa 920-8640, Japan, and ${ }^{4}$ Core Research for Evolutional Science \\ and Technology, JST, Saitama 332-0012, Japan
}

During brain development, various types of neuronal populations are produced from different progenitor pools to produce neuronal diversity that is sufficient to establish functional neuronal circuits. However, the molecular mechanisms that specify the identity of each progenitor pool remain obscure. Here, we show that Wnt signaling is essential for the specification of the identity of posterior progenitor pools in the Drosophila visual center. In the medulla, the largest component of the visual center, different types of neurons are produced from two progenitor pools: the outer proliferation center (OPC) and glial precursor cells (GPCs; also known as tips of the OPC). We found that OPC-type neurons are produced from the GPCs at the expense of GPC-type neurons when Wnt signaling is suppressed in the GPCs. In contrast, GPC-type neurons are ectopically induced when Wnt signaling is ectopically activated in the OPC. These results suggest that Wnt signaling is necessary and sufficient for the specification of the progenitor pool identity. We also found that Homothorax (Hth), which is temporally expressed in the OPC, is ectopically induced in the GPCs by suppression of Wnt signaling and that ectopic induction of Hth phenocopies the suppression of Wnt signaling in the GPCs. Thus, Wnt signaling is involved in regionalization of the fly visual center through the specification of the progenitor pool located posterior to the medulla by suppressing Hth expression.

Key words: Drosophila; GPC; OPC; optic lobe; wingless; Wnt

\section{Significance Statement}

Brain consists of considerably diverse neurons of different origins. In mammalian brain, excitatory and inhibitory neurons derive from the dorsal and ventral telencephalon, respectively. Multiple progenitor pools also contribute to the neuronal diversity in fly brain. However, it has been unclear how differences between these progenitor pools are established. Here, we show that Wnt signaling, an evolutionarily conserved signaling, is involved in the process that establishes the differences between these progenitor pools. Because $\beta$-catenin signaling, which is under the control of Wnt ligands, specifies progenitor pool identity in the developing mammalian thalamus, Wnt signaling-mediated specification of progenitor pool identity may be conserved in insect and mammalian brains.

\section{Introduction}

A wide variety of neurons are generated from neural stem cells, and the neurons establish complex neuronal circuits during brain

\footnotetext{
Received Feb. 28, 2016; revised April 26, 2016; accepted May 9, 2016

Author contributions: T.S. and M.S. designed research; T.S., O.T., T.Y., and R.T. performed research; T.S. analyzed data; T.S. and M.S. wrote the paper.

This work was supported by PRESTO and Core Research for Evolutional Science and Technology from JST, Grantin-Aid for Scientific Research on Innovative Areas and Grant-in-Aid for Scientific Research (B) from MEXT, Sekisui Chemical Grant Program, and Asahi Glass Foundation to M.S. We thank Adi Salzberg, Barry Dickson, Jim Skeath, and Tetsuya Tabata for antibodies and fly strains; Bloomington Stock Center, Vienna Drosophila RNAi Center, and DGRC, Kyoto for fly strains; and Developmental Studies Hybridoma Bank and Asian Distribution Center for Segmentation Antibodies for antibodies.

The authors declare no competing financial interests.

*T.S. and 0.T. contributed equally to this work.

Correspondence should be addressed to Dr. Makoto Sato, Mathematical Neuroscience Unit, Institute for Frontier Science Initiative, Kanazawa University, 13-1 Takaramachi Kanazawa-shi, Ishikawa 920-8640, Japan. E-mail: makotos@staff.kanazawa-u.ac.jp.
}

development to ensure correct brain function. To acquire neuronal diversity, different types of neuronal populations are produced from different progenitor pools. In the mammalian cerebral cortex, excitatory neurons are produced from the radial glial cells located at the ventricular zone of the dorsal telencephalon, whereas inhibitory neurons are produced from medial and caudal ganglionic eminences located at the ventral telencephalon (Anderson et al., 1997; Xu et al., 2004).

Multiple progenitor pools also contribute to neuronal diversity in the developing thalamus in mammals. The large thalamic progenitor pool located at the caudal region is called pTH-C and is the source of glutamatergic neurons; the small thalamic progenitor pool, located at the rostral region, is called pTH-R and is 
the source of GABAergic neurons (Vue et al., 2007; Jeong et al., 2011). Thus, neurons in both the cerebral cortex and thalamus are produced from multiple progenitor pools with different identities. Although extensive studies have revealed that sonic hedgehog, fibroblast growth factor, and $\beta$-catenin signalings play essential roles in the specification of each progenitor pool, a full picture of the underlying molecular mechanisms remains unclear (Kataoka and Shimogori, 2008; Vue et al., 2009; Xu et al., 2010; Flandin et al., 2011; Bluske et al., 2012).

The fly visual center is a good model for neurodevelopmental studies because of many similarities to mammalian neurogenesis. For example, neural stem cells differentiated from neuroepithelial cells produce neural progenitors through asymmetric cell divisions in both fly visual center and mammalian cerebral cortex. The medulla is the largest component of visual center and contains $\sim 100$ types of 40,000 neurons. These medulla neurons are generated from the neuroblasts (NBs), neural stem cell-like progenitor cells, located on the surface of the medulla primordium in a region called the outer proliferation center (OPC). In addition to the OPC, the posterior region of the medulla primordium, which is called the glia precursor cells (GPCs) or the tips of the OPC (Bertet et al., 2014), is also the source of medulla neurons as we and others have recently reported (Bertet et al., 2014; Suzuki et al., 2016a) (see Fig. 1A). The GPCs produce unique types of neurons, called the lamina wide-field neurons whose axons project toward the lamina, the first ganglion in the visual center (Fischbach and Dittrich, 1989; Hasegawa et al., 2011; Tuthill et al., 2013). Thus, the medulla primordium contains two different progenitor pools, the OPC and the GPCs, and they produce distinct types of neurons. However, mechanisms that specify the identity of these progenitor pools have not been clarified.

To understand the mechanisms that distinguish the distinct progenitor pools, we investigated the molecular mechanisms that specify the identity of OPC and GPCs and found that Wnt signaling is essential for GPC specification. When Wnt signaling was suppressed, OPC-type neurons were ectopically observed within the GPC region, suggesting that the posterior progenitors lost their GPC identity; and instead, the cells obtained the OPC identity. We also found that the expression of Homothorax (Hth), which is expressed in the OPC, was suppressed by Wnt signaling and that ectopic activation of Hth transformed GPCs to OPC. The present study demonstrates that Wnt signaling regulates the identity of the neuronal progenitor pool in the fly visual system. Similarly, $\beta$-catenin signaling, which is under the control of Wnt family ligands, specifies the progenitor pool identity in the developing mammalian thalamus (Bluske et al., 2012). Our results show that evolutionarily conserved signaling molecules determine the regional specificity of neural progenitor cells, raising the possibility that this regulatory mechanism is conserved from insects to mammalian brains.

\section{Materials and Methods}

Fly strains. All fly strains were reared on standard Drosophila medium at $25^{\circ} \mathrm{C}$. For all experiments in this study, both male and female fly larvae at wandering late third instar were used. The fly strains used were as follows: hs-flp, FRT82B, ubi-GFP, act>yellow $>$ Gal4 (Ito et al., 1997), UAS-GFP, UAS-CD8GFP, UAS-dicer2, UAS-flp, act>stop>lacZ, omb-Gal4, wgGal4, NP2631 (optix-Gal4), dsh ${ }^{\text {VA153 }}$, Axin, UAS-arm ${ }^{\text {S10 }}, U A S-d s h^{431 B 1}$, UAS-gpi-Dfz2, UAS-pan ${ }^{D N 4}, U A S-d a d, U A S-t k v^{Q 253 D}, h t h^{P 2}, U A S-h t h^{12}$, UAS- $h$ th RNAi ( $h t h^{J F 02733}$ and $h t h^{H M S O 1112}$ ), and robo2 $2^{\text {robo2-HA }}$ (Spitzweck et al., 2010). For the simultaneous suppression of Hth expression and Wnt signaling, the following lines were used: omb-Gal4 UAS-CD8GFP/+; $U_{A S-\text { pan }^{D N 4} / U A S-d i c e r 2 ;}{ }^{D A S}-C D 8 G F P /+$, omb-Gal4 UAS-CD8GFP/+; $U A S-$ pan $^{D N 4} / U A S$-dicer2; UAS- $h t^{J F O 2733} /+$, omb-Gal4 UAS-CD8GFP/+;
UAS-pan ${ }^{D N 4} / U A S$-dicer2; UAS- $h \mathrm{hh}^{\mathrm{HMSO1112} /+}$, omb-Gal4 UAS-

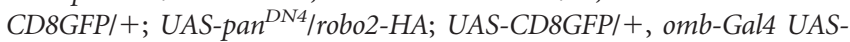
CD8GFP/+; UAS-pan ${ }^{D N 4} /$ robo2-HA; UAS- $h t_{h}^{J F O 2733} /+$, omb-Gal4 UAS-CD8GFP/+; UAS-pan ${ }^{D N 4} /$ robo2-HA; UAS- $h$ th $^{H M S 01112 /+.}$

Clonal analysis. The following genetic crosses and heat shock conditions were used: wg-Gal4 CD8GFP; UAS-flp was crossed to act> stop $>$ lac $Z$ or act $>$ stop $>$ lacZ; UAS-gpi-Dfz2 (see Fig. 3A,B). Axin FRT $82 B$ was crossed to $h s-f l p ; u b i-G F P M$ FRT $82 B\left(37^{\circ} \mathrm{C}, 60 \mathrm{~min}\right.$ at first or second instar larva; see Fig. 5A,B). hs-flp; act>yellow $>$ Gal4 was crossed to $U A S-w g\left(34^{\circ} \mathrm{C}, 30 \mathrm{~min}\right.$ at first or second instar larva; see Fig. $5 C-E)$. robo2 ${ }^{\text {robo2-HA }}$; Axin FRT82B was crossed to hs-flp; ubi-GFP FRT82B $\left(37^{\circ} \mathrm{C}, 60 \mathrm{~min}\right.$ at first or second instar larva; see Fig. $\left.6 E, F\right)$. robo2 ${ }^{\text {robo2-HA}}$; $h t^{P 2}$ FRT82B were crossed to $h s-f l p ; u b i-G F P$ M FRT82B $\left(37^{\circ} \mathrm{C}, 60 \mathrm{~min}\right.$ at first or second instar larva; see Fig. $\left.6 \mathrm{G}\right)$.

Immunohistochemistry. Immunohistochemistry was performed as described previously (Hasegawa et al., 2011). The following primary antibodies were used: guinea pig anti-Bsh (1:1600; Hasegawa et al., 2011), guinea pig anti-Dll (1:2000; Richard Mann, Columbia University), rat anti-Drf (1:3000; Hasegawa et al., 2011), rabbit anti-Hth (1:1000; Kurant et al., 1998), rabbit anti-Lim1 (1:500; James Skeath, Washington University), rabbit anti-Robo2 (1:50; Rajagopalan et al., 2000), mouse anti-LacZ (1:250; Promega), chick anti-LacZ (1:1000; Abcam), rabbit anti-HA (1: 1500; Cell Signaling Technology), and mouse anti-GFP (1:400; Clontech). The following monoclonal antibodies were obtained from Developmental Studies Hybridoma Bank: mouse anti-Eya (1:8), mouse anti-Repo (1:10), rat anti-Ncad (1:20), mouse anti-Fas3 (1:10), and rat anti-Elav (1:50). The secondary antibodies used were anti-guinea pig Cy5, anti-guinea pig Alexa-467, anti-mouse Cy3, anti-mouse Cy5, antimouse FITC, anti-rat Dylight 649, anti-rat Cy5, anti-rabbit FITC, antichick Cy3, anti-chick Alexa-647 (Jackson ImmunoResearch Laboratories), and anti-rabbit Alexa-546 (Invitrogen). Confocal images were acquired using Zeiss LSM510 and LSM880 and were processed using Zeiss ZEN image browser. The number of Eya ${ }^{+}$neurons or glial cells was counted along a single optical section, which was selected according to the neuropile structure visualized by Ncad staining (see Fig. $7 B)$. Mean and SD values were compared and statistically tested by unpaired two-tailed Student's $t$ test (equal or unequal variances).

\section{Results}

\section{Wnt signaling is required for the production of GPC-} type neurons

In the developing medulla primordium, the OPC-NBs produce medulla neurons with a radial and linear orientation toward the center of the primordium, and their neuronal types are specified in a birth order-dependent manner ( $\mathrm{Li}$ et al., 2013; Suzuki et al., 2013). Thus, the larval medulla primordium is subdivided into concentric zones that are characterized by the expression of conserved transcription factors, namely, Hth, Brain-specific homeobox (Bsh), Runt (Run), and Drifter (Drf) (Hasegawa et al., 2011; Sato et al., 2013; Suzuki and Sato, 2014) (Fig. 1A). The $\mathrm{Hth}^{+}$domain is further subdivided into two distinct domains characterized by the expression of Bsh: the outer domain is $\mathrm{Hth}^{+} / \mathrm{Bsh}^{+}$, and the inner domain is $\mathrm{Hth}^{+} / \mathrm{Bsh}^{-}$. The outer $\mathrm{Hth}^{+} / \mathrm{Bsh}^{+}$domain is occupied by neurons derived from the OPC, whereas the inner $\mathrm{Hth}^{+} / \mathrm{Bsh}^{-}$domain contains neuronal populations derived from the GPCs (Suzuki et al., 2016a) (Fig. 1A). These GPCtype neurons are specifically defined by the expression of Eya.

To examine molecular mechanisms that regulate the production of GPC-type neurons, we focused on roles of Wnt signaling because $w g$-Gal4 is specifically expressed in the GPCs (Fig. $1 B, C$ ) (Kaphingst and Kunes, 1994). Eya ${ }^{+}$neurons were almost completely lost, and the wg-Gal4-expressing region was variably reduced when Wnt signaling was suppressed in the GPCs by the induction of a dominant negative form of the $\mathrm{Wg}$ receptor, Dfrizzled2 (Dfz2 $\left.{ }^{\mathrm{DN}}\right)$, under the control of $w g$-Gal4 (Figs. $1 D, 7 E$; 
A

\section{lateral view}

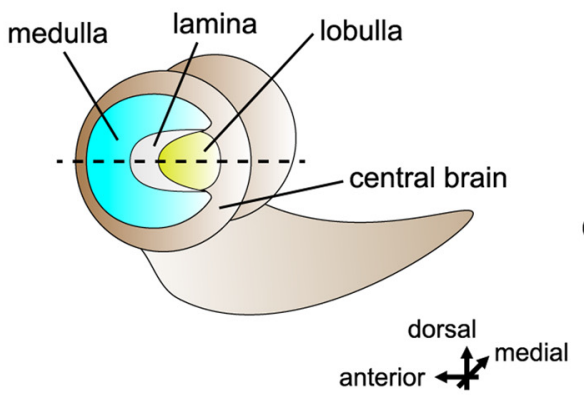

\section{horizontal view}

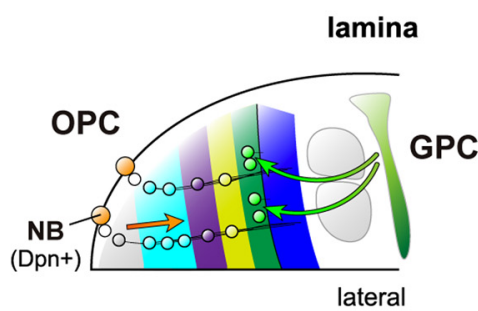

$\uparrow$ posterior

B

lateral view
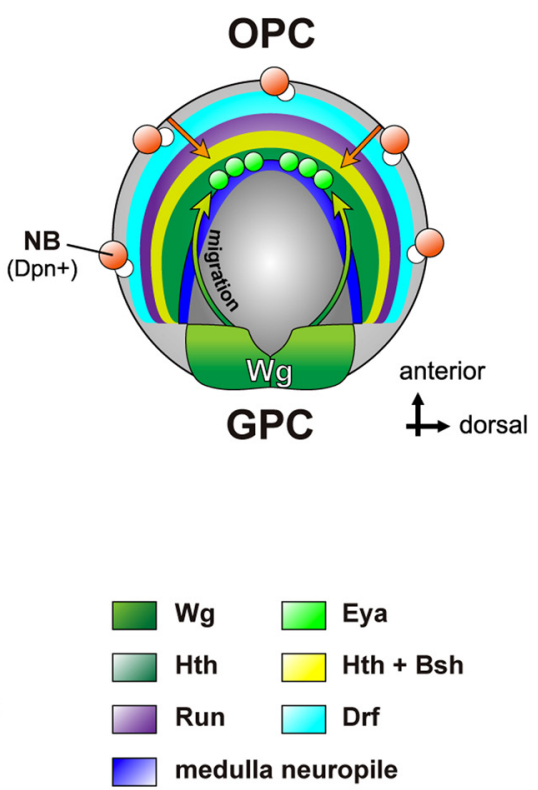

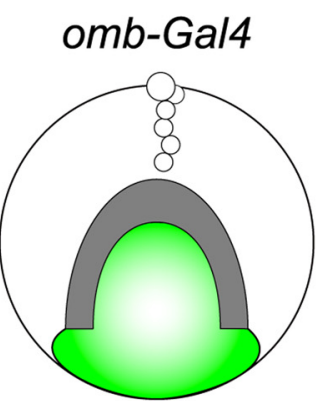

wg-Gal4

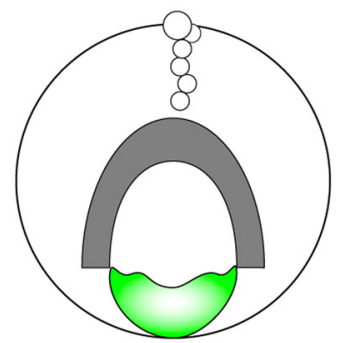

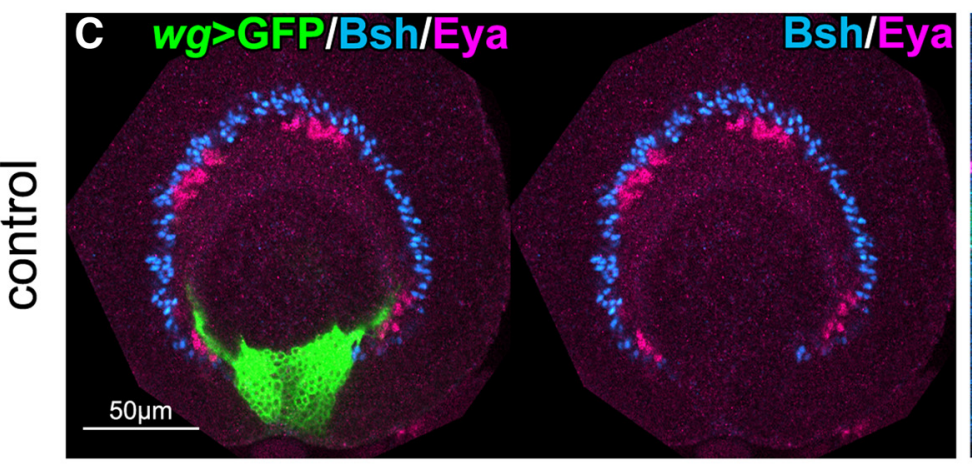
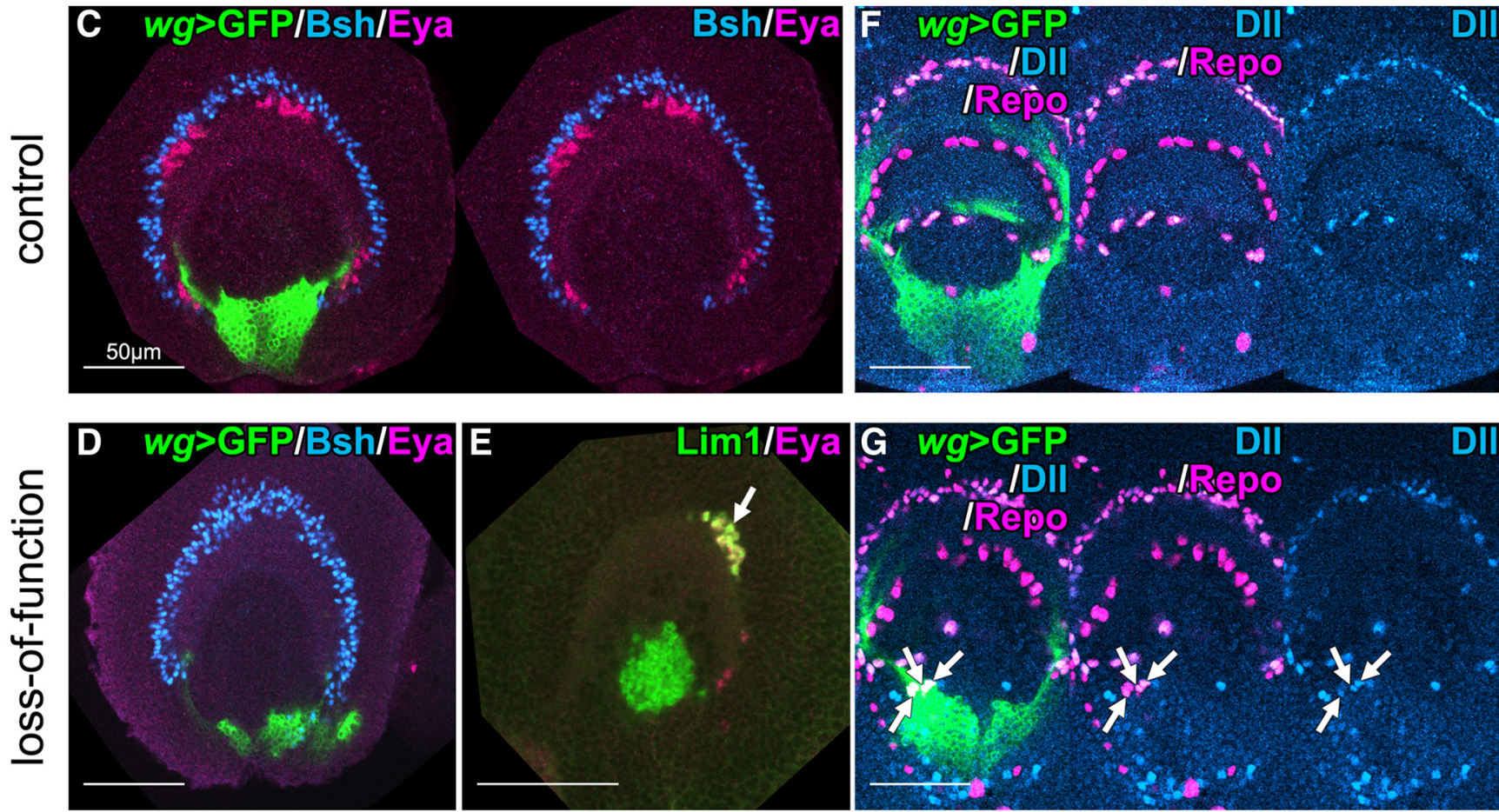

Figure 1. Wnt signaling is required for the production of GPC-type neurons. A, Schematics of the larval CNS and medulla primordium in lateral and horizontal views. OPC-NBs produce medulla neurons in a linear and radial orientation toward the center of medulla (orange arrows). NBs are also found in the GPC region located at the posterior region of the medulla (light green). GPC-type neurons migrate tangentially and are placed in the innermost region of medulla cortex. $\boldsymbol{B}$, Schematics of the expression patterns of omb-Gal4 (top) and wg-Gal4 (bottom) in lateral views. All of the following images are lateral views of the medulla in wandering late third instar larva (top, anterior; right, dorsal). C, D, OPC-type Bsh ${ }^{+}$neurons (blue), GPC-type Eya ${ }^{+}$neurons (magenta), and wg-Gal4 ${ }^{+}$GPCs (green) are visualized. $\boldsymbol{D}$, Expressing dominant negative form of Dfz2 under the control of $w g$-Gal4, Eya ${ }^{+}$neurons were completely lost compared with the control (C).E, The ventral GPC-neurons were completely lost in dsh mutant, although the dorsal GPC-neurons (magenta represents Eya ${ }^{+}$; and green represents Lim $1^{+}$) were still observed (white arrow). $F$, MNGs (magenta represents Repo ${ }^{+}$; and blue represents $\mathrm{DII}^{+}$) and $w g-G a l 4^{+} \mathrm{GPCs}$ (green) are visualized. G, MNGs were ectopically induced (white arrows) in the GPC region by the dominant negative form of Dfz2 compared with the control $(\boldsymbol{F})$. 

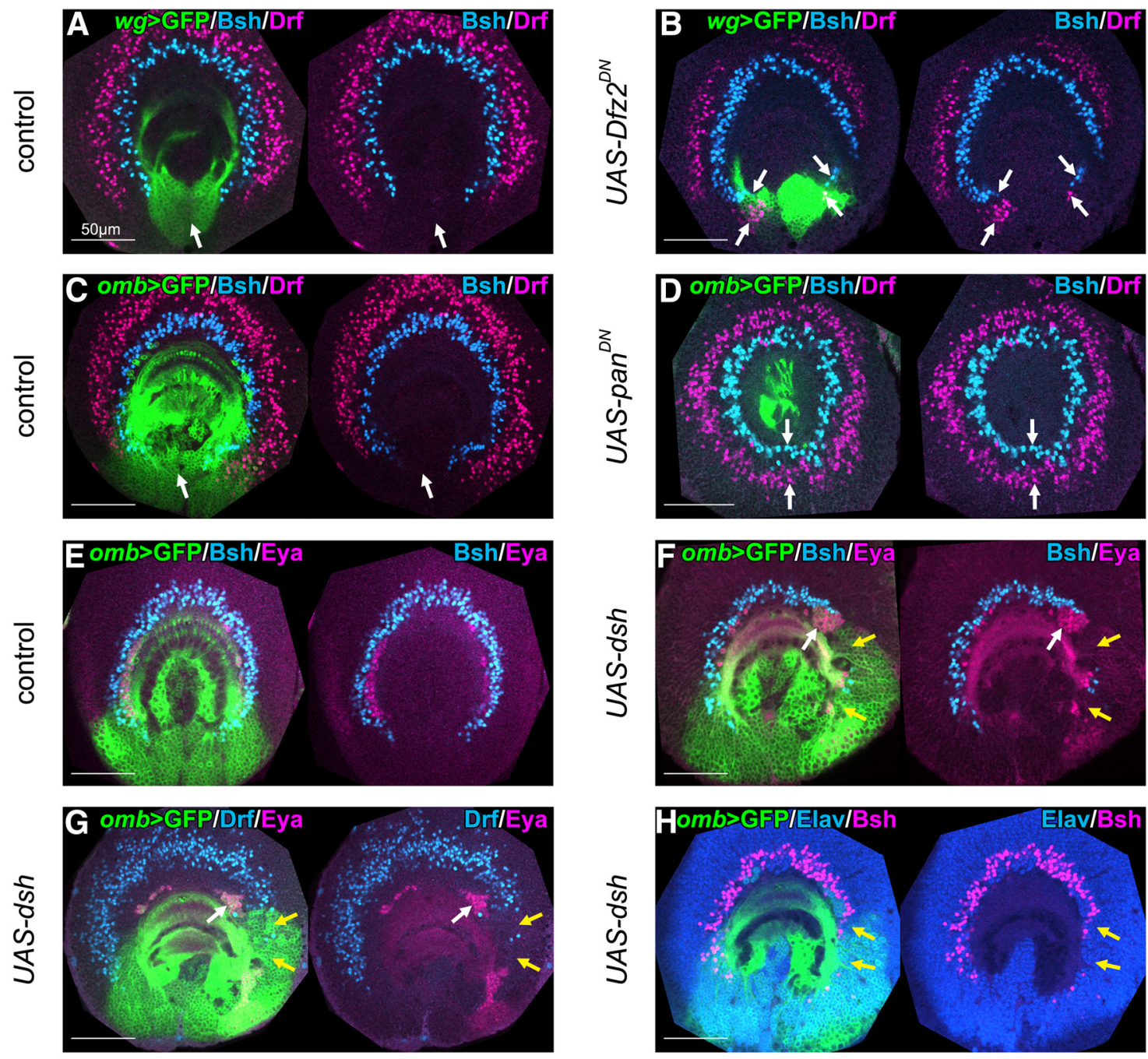

Figure 2. Wnt signaling represses the formation of OPC-type neurons. $A, C, O P C$-type neurons (blue represents Bsh ${ }^{+}$; and magenta represents Drf ${ }^{+}$) were not observed in the GPC region (green, white arrows) in control larvae. $\boldsymbol{B}$, Inducing dominant negative form of Dfz2 under the control of $w g$-Gal4, OPC-type neurons (blue represents Bsh ${ }^{+}$; and magenta represents Drf ${ }^{+}$) were ectopically observed (white arrows). $\boldsymbol{D}$, Inducing dominant negative form of Pan under the control of omb-Gal4, OPC-type neurons (blue represents Bsh ${ }^{+}$; and magenta represents Drf ${ }^{+}$) were ectopically observed (white arrows) forming complete circles. $\boldsymbol{E}$, Eya ${ }^{+} \mathrm{GPC}$-type neurons (magenta) and Bsh ${ }^{+}$OPC-type neurons (blue) were normally observed in control larvae. $\boldsymbol{F}$ - $\boldsymbol{H}$, Expressing dsh under the control of omb-Gal4, OPC-type Bsh ${ }^{+}$(blue in $\boldsymbol{F}$; and magenta in $\boldsymbol{H}$ ) and Drf ${ }^{+}$neurons (blue in $\boldsymbol{G}$ ) were partially lost $\left(\boldsymbol{F}, \boldsymbol{G}\right.$, yellow arrows). $\mathrm{GPC}$-type Eya ${ }^{+}$neurons (magenta) were increased $(\boldsymbol{F}, \mathbf{G}$, white arrows), whereas Elav expression was normal (blue in $\boldsymbol{H}$; yellow arrows).

$n=30 / 35)$. Likewise, ventral Eya ${ }^{+}$cells were lost, and only dorsal $\mathrm{Lim} 1^{+} / \mathrm{Eya}^{+}$cells remained in the mutant for dishevelled $\left(d s h^{V A 153}\right)$, one of the essential components of the Wnt signaling pathway (Fig. $1 E$, white arrow; $n=27 / 39$ ).

In addition to the production of Eya ${ }^{+}$neurons, GPCs produce glial cells for the lamina, the first ganglion that processes visual information. Surprisingly, we found that the suppression of Wnt signaling in GPCs significantly increased the production of glial cells located in the medulla primordium. Medulla neuropile glia (MNG) are generated from the OPCNBs (Colonques et al., 2007; Soustelle and Giangrande, 2007; Hasegawa et al., 2011; Li et al., 2013; Suzuki et al., 2016b) and were rarely observed within the GPC region in the control (Fig. $1 F ; 2 \pm 0.7$ Repo $^{+}$cells). Suppression of Wnt signaling in the GPCs by the induction of Dfz2 ${ }^{\mathrm{DN}}$ under the control of $w g$-Gal4 caused the generation of ectopic MNG-like glial cells (Fig. $1 G$, white arrows; $n=10 / 16 ; 12.5 \pm 2.0$ Repo $^{+}$cells, $p<$ 0.001 compared with Fig. $1 F$ ), as visualized by Dll and Repo (Suzuki et al., 2016b), in addition to the significant reduction in the number of Eya ${ }^{+}$neurons (Figs. $1 D, 7 E$ ).
Wnt signaling represses the formation of OPC-type neurons The above results raise the possibility that Wnt signaling defines the fate of medulla precursor cells to differentiate into GPC-type progeny, and we further assumed that the disruption of Wnt signal transduction caused failure in the formation of the posterior region of the medulla primordium and, consequently, the posterior region obtained the anterior identity. Is the GPC region transformed into OPC by the suppression of Wnt signaling? To answer this question, we examined the expression patterns of Bsh and Drf, markers for OPC-type neurons (Fig. 1A), when Wnt signaling was suppressed. In the control brains, $\mathrm{Bsh}^{+}$and $\mathrm{Drf}^{+}$neurons were arranged concentrically across the anterior and dorsoventral regions, but they were never observed in the posterior region (Fig. 2A, white arrows). $\mathrm{Bsh}^{+}$and $\mathrm{Drf}^{+}$neurons were ectopically observed in the $\mathrm{wg}$-Gal4 ${ }^{+}$posterior region when Wnt signaling was suppressed by the induction of $D f z 2^{D N}$ under the control of $w g$-Gal4 (Fig. $2 B ; n=35 / 44$, white arrows). A similar phenotype was observed in $d s h$ mutant and $w g^{t s}$ homozygous third instar larvae kept at restrictive temperature $\left(30^{\circ} \mathrm{C}\right)$ for $48 \mathrm{~h}$ 

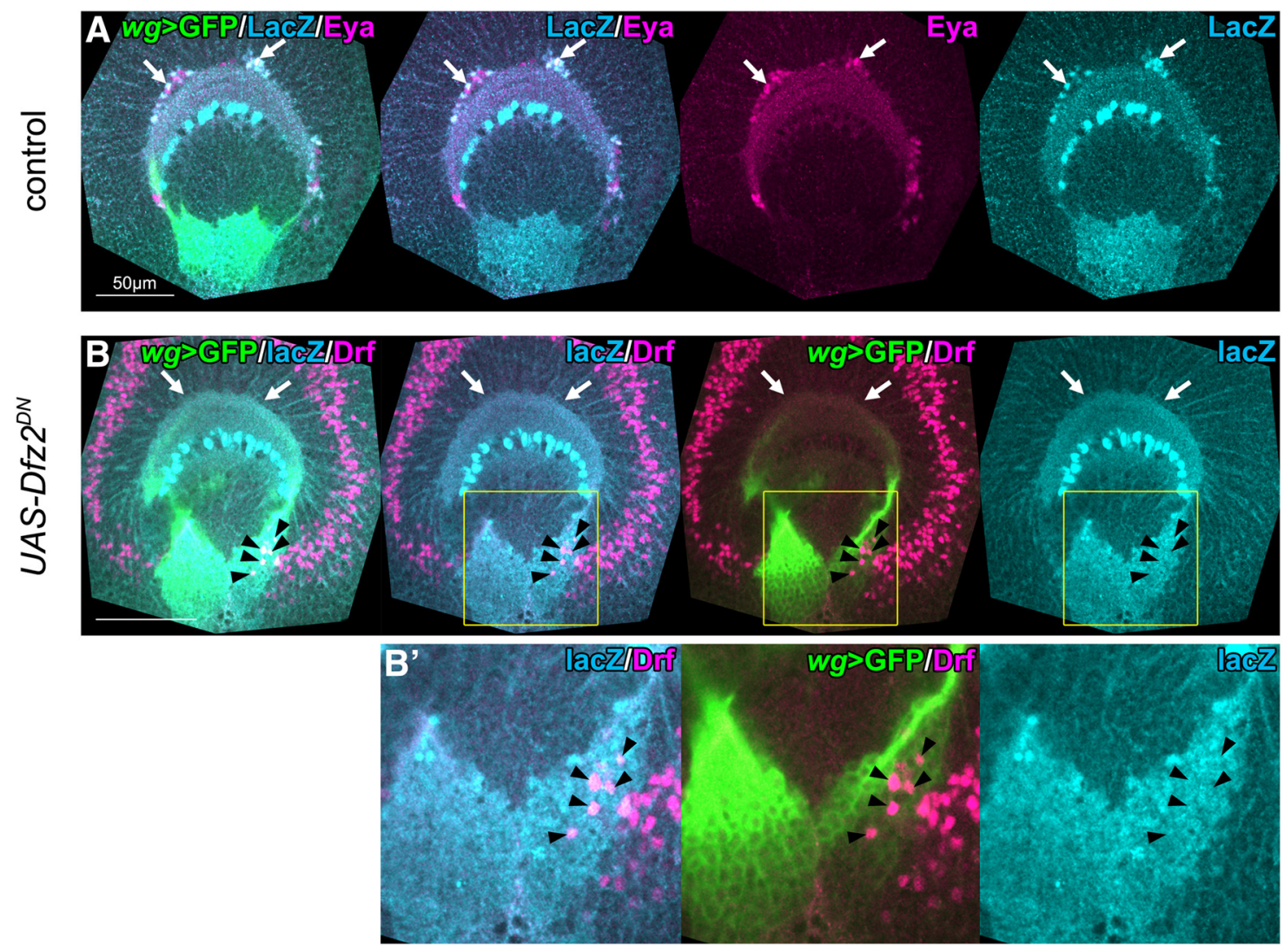

Figure 3. GPCs produce OPC-type neurons when Wnt signaling is suppressed. $A$, The lineages of GPCs were labeled by LacZ under the control of wg-Gal4 UAS-CD8GFP (green), UAS-flp, and $a c t>$ stop $>$ lacZ. LacZ expression (blue) was detected in a subset of Eya ${ }^{+}$cells (magenta, arrows). $\boldsymbol{B}$, When the dominant negative form of Dfz2 was expressed under the control of wg-Gal4, lacZ expression was not detected in the innermost region of the medulla cortex (white arrows). LacZ (blue) and Drf (magenta) double-positive neurons were observed within GPCs (black arrowheads). $\boldsymbol{B}^{\prime}$, Magnified images of the areas indicated by yellow squares in $\boldsymbol{B}$.

(data not shown; $n=8 / 16$ and $n=6 / 6$, respectively). Surprisingly, we observed that $\mathrm{Bsh}^{+}$and $\mathrm{Drf}^{+}$neurons were arranged completely circularly when Wnt signaling was disrupted by the induction of a dominant negative form of Pangolin $\left(\mathrm{Pan}^{\mathrm{DN}}\right)$, the Drosophila homolog of Tcf, under the control of omb-Gal4 (Fig. $2 D$, white arrows, $n=24 / 36$ ). Notably, there was no gap in the posterior region of the $\mathrm{Bsh}^{+}$and $\mathrm{Drf}^{+}$ concentric zones (Fig. 2D, white arrows). This phenotype showed a sharp contrast to the incomplete circular concentric zones with a gap in the posterior region of wild-type brains (Fig. 2C, white arrows), suggesting that the posterior region of the medulla primordium was no longer the GPCs in the absence of Wnt signaling.

Although Eya ${ }^{+}$neurons were significantly decreased by the suppression of Wnt signaling (Fig. 1C,D), activation of Wnt signaling within GPC region did not show any defect when the constitutively active form of Armadillo (Arm), the Drosophila homolog of $\beta$-catenin, was induced (data not shown; $n=13 / 13$ ). However, GPC-type neurons were induced by ectopically activating Wnt signaling outside the GPC region using omb-Gal4. When Dsh was induced, a large cluster of Eya ${ }^{+}$neurons was observed (Fig. 2E-G, see Fig. 7E; Fig. 2F, G, white arrows); instead, Bsh ${ }^{+}$ and $\mathrm{Drf}^{+}$neurons were significantly reduced in the region adjacent to GPCs (Fig. 2E-G; Fig. $2 F, G$, yellow arrows; Fig. $2 F, n=$ 17/19; Fig. $2 G, n=10 / 12$ ). Although Bsh and Drf expression was lost, Elav expression was normal (Fig. $2 H$, yellow arrows, $n=$ 9/10), suggesting that neurons of different identities occupied this region. Notably, the increase in Eya ${ }^{+}$neurons and the loss of
$\mathrm{Bsh}^{+}$and $\mathrm{Drf}^{+}$neurons were exclusively observed in the dorsal region, but not in the ventral region of the medulla primordium (Fig. $2 F-H$ ). The dorsal region may be more sensitive to the increase in Wnt signaling, or ectopic Wnt activity may be blocked in the ventral region by unidentified mechanisms.

OPC-type neurons were observed within the GPC region after the suppression of Wnt signaling (Fig. 2), implying that the GPCs were transformed into the OPC and produced Bsh ${ }^{+}$ and $\mathrm{Drf}^{+}$neurons. To examine whether the $\mathrm{Bsh}^{+}$and $\mathrm{Drf}^{+}$ neurons were produced from the Wnt signaling-deficient GPCs, we conducted FLP-FRT-based lineage analysis under the control of $w g$-Gal4 UAS-flp, and the lineages of the GPCs $\left(w g-\mathrm{Gal}^{+}\right)$were constitutively labeled by LacZ expression under the control of $a c t>$ stop $>l a c Z$ as we reported previously (Suzuki et al., 2016a). In the control, the GPCs themselves and $\mathrm{Eya}^{+}$neurons labeled with LacZ were observed (Fig. 3A, white arrows) (Suzuki et al., 2016a). However, such lac ${ }^{+}$cells were not observed in the innermost region of the medulla cortex when Wnt signaling was suppressed (Fig. 3B; white arrows indicate the loss of $\mathrm{LacZ}^{+}$cells). Instead, $\mathrm{LacZ}^{+} / \mathrm{Drf}^{+}$neurons were observed within the GPC region (Fig. $3 B$, black arrowheads). These results suggest that the posterior region of the medulla primordium failed to obtain the GPC identity and instead obtained the OPC identity to produce OPC-type neurons via the inhibition of Wnt signaling. Therefore, Wnt signaling has essential roles in the specification of these progenitor identities. 

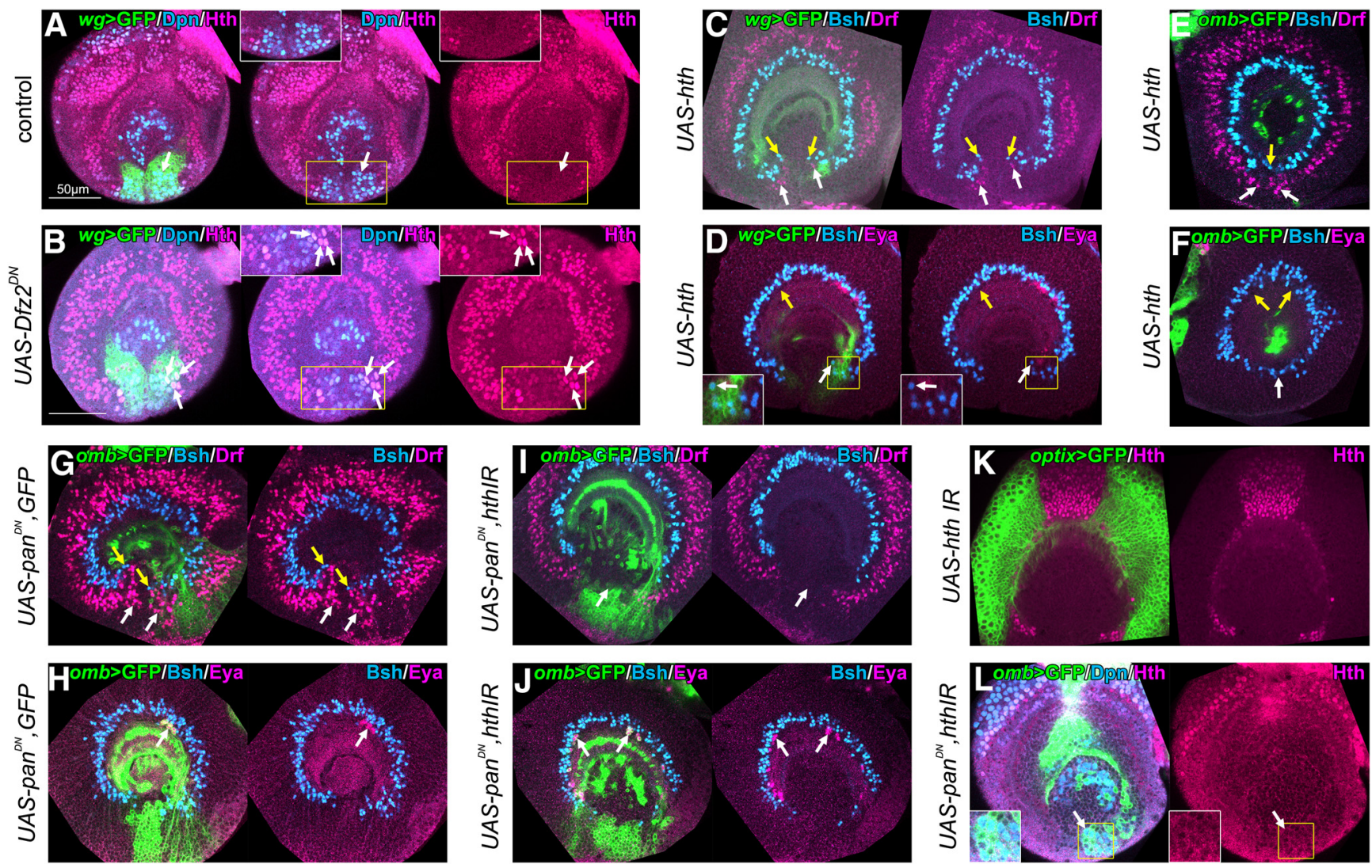

Figure 4. Wnt signaling transforms OPC to GPCs by repressing Hth expression. $A$, Hth (magenta) is not expressed in NBs (Dpn ${ }^{+}$, blue) in the GPC region (green) in control larvae (white arrows), $B$, Expressing dominant negative form of Dfz2 under the control of wg-Gal4, Hth (magenta) was ectopically expressed in NBs in GPC region (Dpn ${ }^{+}$, blue; white arrows). C, Expressing hth under the control of wg-Gal4, Bsh ${ }^{+}$(blue, yellow arrows) and Drf ${ }^{+}$(magenta, white arrows) neurons were ectopically formed within GPC region compared with the control in Figure $2 A$. D, Inducing hth expression, ventral Eya ${ }^{+}$(magenta) neurons were lost (yellow arrow), and Bsh ${ }^{+}$(blue) neurons were ectopically formed (white arrow) compared with the control in Figure $1 C$. $E$, Inducing Hth expression under the control of omb-Gal4, Bsh ${ }^{+}$(blue, yellow arrows) and Drf $^{+}$(magenta, white arrows) neurons were ectopically observed forming complete circles compared with the control in Figure 2C.F, Inducing Hth expression, Eya ${ }^{+}$neurons (magenta) were completely lost (yellow arrows) and Bsh ${ }^{+}$neurons (blue, white arrow) were ectopically observed compared with the control in Figure 2E. $\mathbf{G}, \boldsymbol{H}$, Dominant negative form of Pan under the control of omb-Gal4 induced ectopic Bsh ${ }^{+}$(blue in $\boldsymbol{G}$ ) and Drf ${ }^{+}$(magenta in $\boldsymbol{G}$ ) neurons within GPC region ( $\boldsymbol{G}$, yellow and white arrows, respectively). Eya ${ }^{+}$neurons were reduced (magenta in $\boldsymbol{H}$; white arrows). $\boldsymbol{I}$, Simultaneously suppressing Hth expression and Wnt signaling by expressing dominant negative form of Pan and $\boldsymbol{H}$ th $\mathrm{RNAi}$ ectopic Bsh ${ }^{+}$(blue) and $\mathrm{Drf}^{+}$(magenta) neurons were disappeared (white arrows; compare with $\mathbf{G}$ ). J, The number of Eya ${ }^{+}$neurons (magenta) was not rescued (white arrows) by simultaneous suppression of Hth expression and Wnt signaling (compare with $\boldsymbol{H}$ ). $\boldsymbol{K}$, Hth expression was completely suppressed by induction of Hth RNAi under the control of optix-Gal4. $\boldsymbol{L}$, Hth (magenta) expression in NBs (Dpn ${ }^{+}$, blue) was suppressed (white arrows) by Hth RNAi and Wnt signaling inhibition. Insets, Magnified images of the areas indicated by yellow squares. All of the images, except $\boldsymbol{B}$, are at the same magnification shown by a scale bar in $\boldsymbol{A}$.

\section{Wnt signaling transforms OPC to GPCs by repressing Hth expression}

The OPC-NBs produce various types of medulla neurons, and the neuronal types are specified by the transcription factors that are expressed transiently in the OPC-NBs (temporal factors), as reported previously (Li et al., 2013; Suzuki et al., 2013). The temporal factors are also involved in the specification of neuronal types in the GPCs, but the set of temporal transcription factors in the GPCs is different from that in OPC-NBs (Bertet et al., 2014). $\mathrm{Hth}$ is expressed in the youngest OPC-NBs and specifies $\mathrm{Hth}^{+}$/ $\mathrm{Bsh}^{+}$neurons (Li et al., 2013; Suzuki et al., 2013); however, it is not expressed in the GPCs (Bertet et al., 2014; Suzuki et al., 2016a). If the GPC region is transformed into the OPC via the suppression of Wnt signaling, the set of temporal factors in the GPCs can also be changed to those of the OPC. Therefore, we examined the expression pattern of Hth in the Wnt signalingdeficient GPCs. As previously described, Hth was not expressed in the GPC-NBs (visualized with wg-Gal4 and anti-Dpn antibody) in control larvae (Fig. $4 A$, white arrows; $n=10 / 10$ ) (Bertet et al., 2014). Dpn is a marker for NBs in the OPC and GPC regions. $\mathrm{Hth}^{+} \mathrm{NBs}$ were observed in the GPC region when Wnt signaling was suppressed (Fig. $4 B$, white arrows; $n=26 / 44$ ). This result also suggests that the GPC region was transformed into the OPC in the absence of Wnt signaling.

We next determined the significance of $\mathrm{Hth}$ in the transformation of GPC region to OPC upon the loss of Wnt signaling. To understand the role of $\mathrm{Hth}$, we ectopically induced $\mathrm{Hth}$ in the GPCs under the control of $w g$-Gal4 and examined the distributions of Eya ${ }^{+}, \mathrm{Bsh}^{+}$, and Drf ${ }^{+}$neurons. We found that $\mathrm{Bsh}^{+}$and $\mathrm{Drf}^{+}$neurons appeared ectopically in the posterior region (Fig. $4 C$; yellow arrows indicate ectopic Bsh ${ }^{+}$neurons, $n=25 / 26$; white arrows indicate ectopic Drf ${ }^{+}$neurons, $\left.n=8 / 9\right)$, and Eya ${ }^{+}$ neurons were significantly decreased (Figs. $4 D$, $7 E$; yellow arrows indicate the loss of $\mathrm{Eya}^{+}$neuron, and white arrows indicate ectopic $\mathrm{Bsh}^{+}$neurons in $\left.4 D ; n=13 / 17\right)$. Notably, ventral Eya ${ }^{+}$ neurons were preferentially lost, and dorsal neurons remained normal after the induction of Hth by $w g$-Gal4 (Fig. $4 D ; n=9 / 17$ ). These phenotypes were quite similar to those observed upon Wnt signaling suppression (Figs. $1 D, 2 B$ ). We also examined the effects of ectopic Hth induction under the control of omb-Gal4 and observed the completely circular arrangement of $\mathrm{Bsh}^{+}$and $\mathrm{Drf}^{+}$ neurons (Fig. 4E; yellow and white arrows indicate ectopic Bsh ${ }^{+}$ 

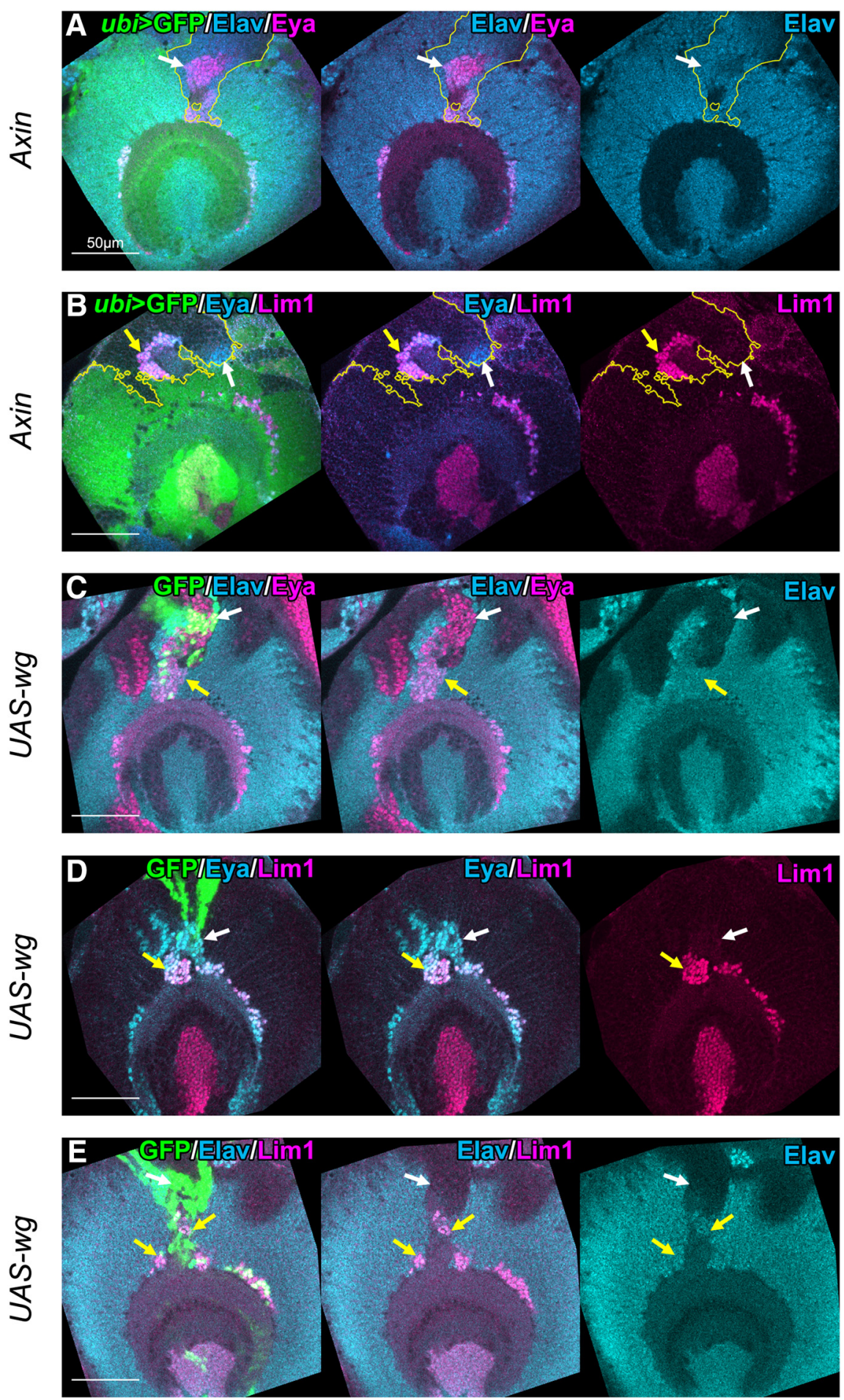

Figure 5. Wnt signaling induces ectopic GPC-type neurons in the anterior region of $O P C$. Clones generated in the anterior region of OPC were examined. $A, B$, Axin mutant clones are labeled by the absence of GFP signals (green). $A$, Eya ${ }^{+}$(magenta)/Elav ${ }^{+}$(blue) neurons were ectopically observed in the clones (white arrows). $\boldsymbol{B}$, Lim $1^{+}$(magenta)/Eya ${ }^{+}$(blue) cells (yellow arrows) and Lim $1^{-}$/Eya ${ }^{+}$cells (white arrows) appeared ectopically in the clones. $\mathbf{C}-\boldsymbol{E}$, Clones expressing $w g$ are labeled by GFP (green). $\boldsymbol{C}$, Eya ${ }^{+}$(magenta)/Elav ${ }^{+}$(blue) neurons were ectopically observed around the clones (yellow arrows). $\boldsymbol{D}, \mathrm{Lim}^{+}{ }^{+}$(magenta)/Eya ${ }^{+}$(blue) cells (yellow arrows) and $\operatorname{Lim} 1^{-} /$Eya ${ }^{+}$cells (white arrows) appeared ectopically around the clones. $E$, Lim $1^{+}$(magenta)/Elav ${ }^{+}$(blue) neurons were observed ectopically around the clones (yellow arrows). $\boldsymbol{C}, \boldsymbol{E}$, Neuronal differentiation was occasionally suppressed, as indicated by white arrows.

and $\mathrm{Drf}^{+}$neurons, respectively, $n=41 / 42$; complete-circular arrangement of $\mathrm{Bsh}^{+}$and $\mathrm{Drf}^{+}$neurons, $\left.n=14 / 42\right)$. In this case, both ventral and dorsal Eya ${ }^{+}$neurons were lost (Fig. 4F; yellow arrows indicate complete loss of Eya ${ }^{+}$neurons; $\left.n=19 / 24\right)$; therefore, the induction of Hth completely mimicked the suppression of Wnt signaling in the GPCs.

Is Hth involved downstream of Wnt signaling? To answer this question, we examined the genetic interaction between Hth and Wnt signaling. In the control flies (omb-Gal4 UAS-CD8GFP/+; UASpan $^{D N 4} / U A S$-dicer2; UAS-CD8GFP/+), $\mathrm{Bsh}^{+}$and $\mathrm{Drf}^{+}$neurons were ectopically observed within the GPC region (Fig. 4G; yellow and white arrows indicate ectopic $\mathrm{Bsh}^{+}$and Drf ${ }^{+}$neurons, respectively; $n=$ 28/33) and $\mathrm{Eya}^{+}$neurons were completely lost $(n=9 / 38)$ or significantly reduced (Figs. 4H, 7E; white arrows indicate the reduction of Eya ${ }^{+}$neurons in Fig. $4 \mathrm{H}$, $n=29 / 38$ ). For loss-of-function analysis of Hth, we used two independent UASRNAi lines $\left(h t h^{J F 02733}\right.$ and $h t h^{H M S 01112}$ ), which completely suppressed Hth expression as visualized by anti-Hth antibody (Fig. $4 K$ ). When hth RNAi and Wnt signaling suppression was simultaneously induced, neither Bsh ${ }^{+}$nor $\mathrm{Drf}^{+}$neurons were ectopically observed in the GPC region (Fig. $4 I$, white arrows; $h t h^{J F 02733}, n=$ 23/33; $\left.h t^{H M S 01112}, n=41 / 65\right)$. Several $\mathrm{Eya}^{+}$neurons were observed in the medulla cortex by the simultaneous suppression of Hth and Wnt signaling (Figs. 4J, $7 E$; Fig. 4J, white arrows; $h t h^{J F 02733}, n=$ 21/39; $h t h^{H M S 01112}, n=24 / 41$ ). Hth expression was undetectable in the GPC region in the same condition (Fig. 4L). These results suggest that the suppression of Hth effectively rescues the ectopic appearance of OPC-type neurons caused by the loss of Wnt signaling and that Hth conveys ectopic OPC formation upon the loss of Wnt signaling. However, the number of Eya ${ }^{+}$GPC-type neurons was not rescued by the coexpression of $h t h$ RNAi (Figs. $4 J, 7 E$ ), implying that Wnt signaling regulates other factors to form GPC-type neurons in addition to the removal of $\mathrm{Hth}$ expression. However, we cannot rule out the possibility that undetectable level of Hth expression suppresses the production of GPC-type neurons (Fig. 4J,L).

\section{Wnt signaling induces ectopic GPC-} type neurons in the anterior region of the OPC

Our results suggest that Wnt signaling is required for the induction of GPCs by blocking anteriorization in the posterior region of the medulla primordium through Hth repression. We then examined whether ectopic activation of Wnt signaling in the anterior region of the medulla primordium induces the GPC-type differentiation. To investigate this possibility, mutant clones of Axin, an inhibitory component of Wnt signaling, were induced in the medulla primor- 

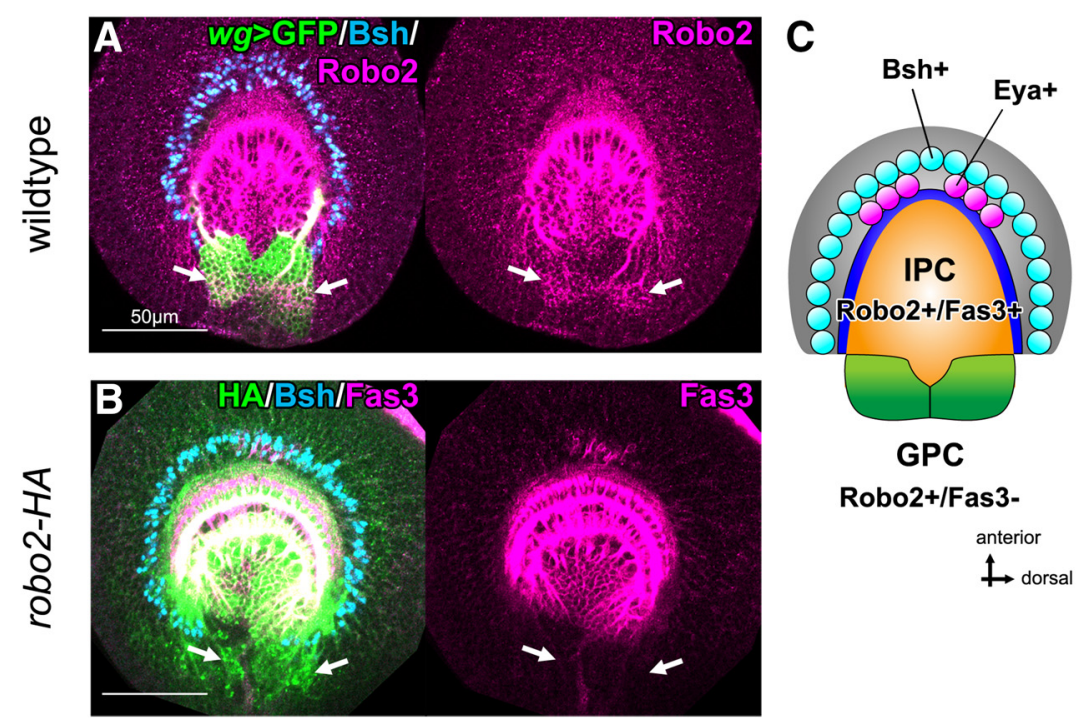

\section{Robo2+/Fas3-}

anterior

$\dashv$ dorsal
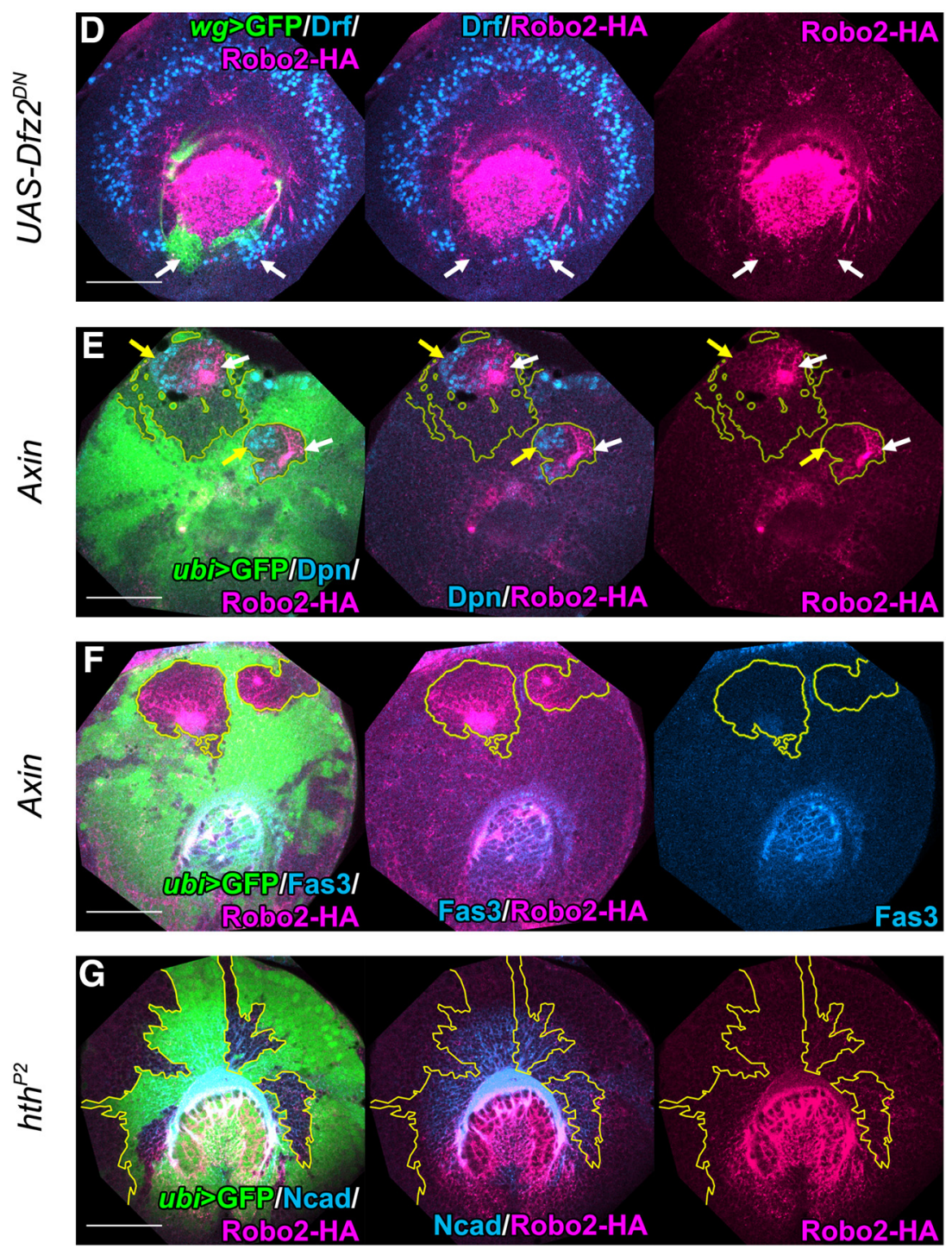

Figure 6. Wnt signaling specifies the GPC identity. $A$, Robo2 (magenta) is expressed in wild-type GPCs visualized by wg-Gal4 UAS-CD8GFP (green, white arrows). $\boldsymbol{B}$, Robo2-HA (green) is expressed in GPCs (white arrows) and IPC (Fas ${ }^{+}{ }^{\text {, }}$, magenta). $\boldsymbol{C}$, A schematic of the expression pattern of Rob02 and Fas 3 in the larval optic lobe. Rob02 and Fas 3 are coexpressed in IPC (orange). Only Rob02 is expressed in GPCs (green). OPC-type Bsh ${ }^{+}$and GPC-type Eya ${ }^{+}$neurons are colored in blue and magenta, respectively. D $_{\text {, }}$ Inducing dominant negative form of Dfz2 under the control of wg-Gal4, Robo2-HA (magenta) expression was lost (white arrows); dium. When Axin mutant clones were created in the anterior region, Eya ${ }^{+}$neurons were ectopically observed (Fig. $5 \mathrm{~A}$, white arrows; $n=29 / 81)$. We also examined whether Lim1 is ectopically induced in Axin mutant clones because Lim1 is specifically expressed in the dorsal half of GPC-type neurons in the medulla primordium (Suzuki et al., 2016a). Lim1 ${ }^{+} / \mathrm{Eya}^{+}$doublepositive neurons were observed in Axin mutant clones (Fig. $5 B$, yellow and white arrows indicate $\mathrm{Lim}^{+} / \mathrm{Eya}^{+}$and $\mathrm{Lim}^{-} /$ Eya $^{+}$neurons, respectively; $n=16 / 41$ ). These results suggest that ectopic activation of Wnt signaling causes ectopic production of GPC-type neurons.

Although artificial activation of Wnt signaling resulted in the ectopic appearance of GPC-type neurons in the anterior region, it is still unclear which type of Wnt ligand is involved in this process. Because wg-Gal4 is specifically expressed in the GPCs, we examined whether ectopic induction of Wg expression mimics the phenotypes of the Axin mutant clones. When Wg-expressing clones were induced in the anterior region, Eya ${ }^{+}$ and $\mathrm{Eya}^{+} / \mathrm{Lim}^{+}$neurons were ectopically observed in and around the clones (Fig. $5 C-E$; Fig. $5 C$, yellow and white arrows indicate $\mathrm{Eya}^{+}$neurons and $\mathrm{Eya}^{+}$nonneuronal cells, respectively, $n=11 / 24$; Fig. $5 D$, yellow and white arrows indicate Lim $1^{+} / \mathrm{Eya}^{+}$and $\mathrm{Lim}^{-} / \mathrm{Eya}^{+}$cells, respectively, $n=7 / 13$; Fig. $5 E$, yellow and white arrows indicate $\operatorname{Lim}^{+}$neurons and non-neuronal cells, respectively, $n=3 / 6$ ). Therefore, it is most likely that $\mathrm{Wg}$ activates Wnt signaling in the GPC region to establish the posterior progenitor pool identity.

\section{Wnt signaling induces GPC identity}

Because GPC-type neurons were induced by the ectopic activation of Wnt signaling, we next examined whether ectopic GPCs are also induced in the anterior region of the medulla primordium. Thus far, GPCs have been visualized with the expression of $w g$-Gal4. Additionally, we found that Robo2, a member of the axon guidance molecules, is specifically expressed in the GPC region (Fig. 6A, white arrows). Although Robo2 is also expressed in the inner proliferation center (IPC) (Suzuki et al., 2016b), which is the source of lobula

\section{$\leftarrow$}

and instead, Drf ${ }^{+}$(blue) cells were ectopically observed (white arrows) in the posterior region. $\boldsymbol{E}, \boldsymbol{F}$, In Axin mutant clones, Robo2-HA expression (white arrows) was ectopically observed in the anterior region of OPC. Robo2-HA coexpresses $\mathrm{Dpn}^{+}$(blue, $\boldsymbol{E}$, yellow arrows) without Fas3 expression (blue, F). G, Robo2-HA (magenta) was not induced in hth mutant clones labeled by the absence of GFP (green). 

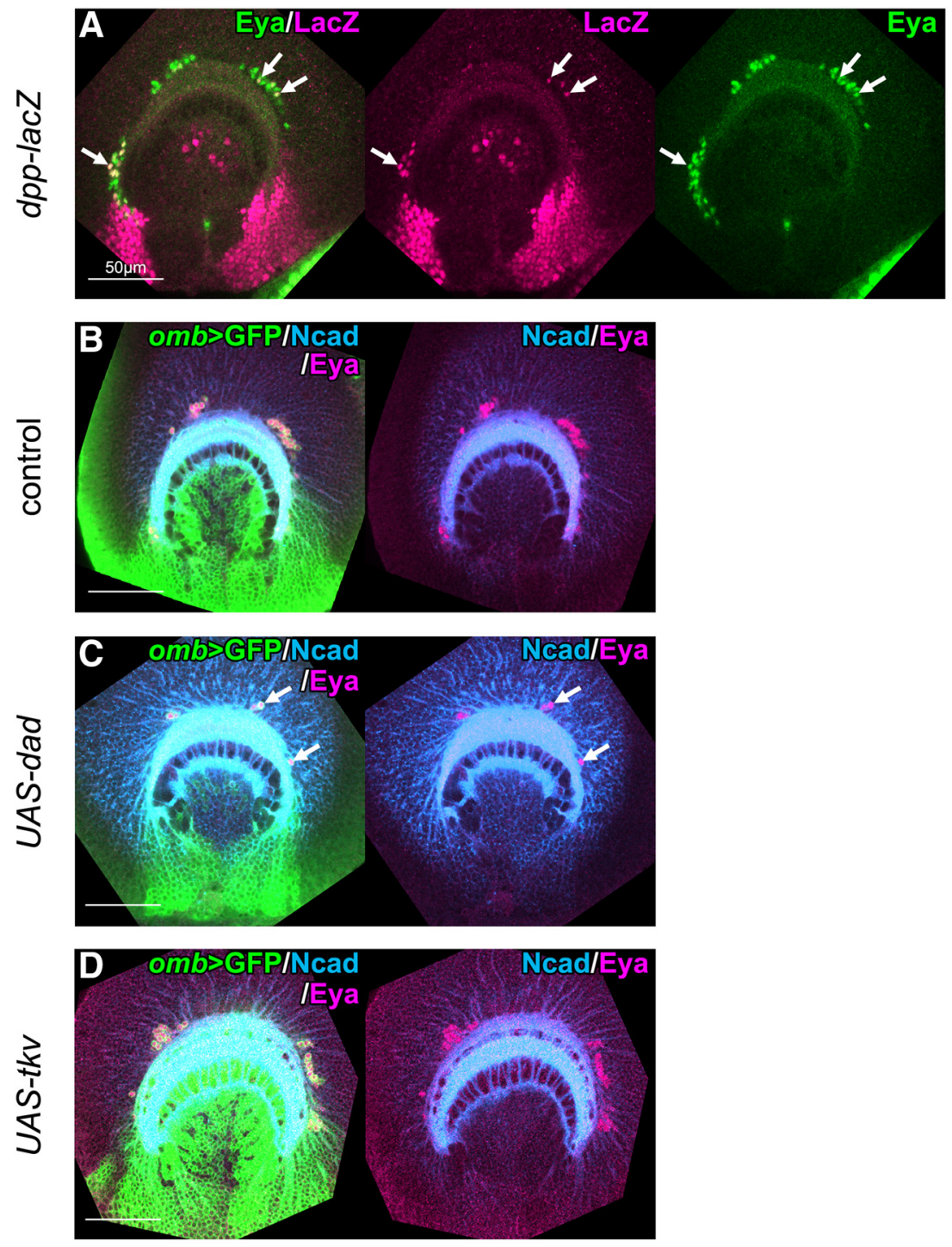

E

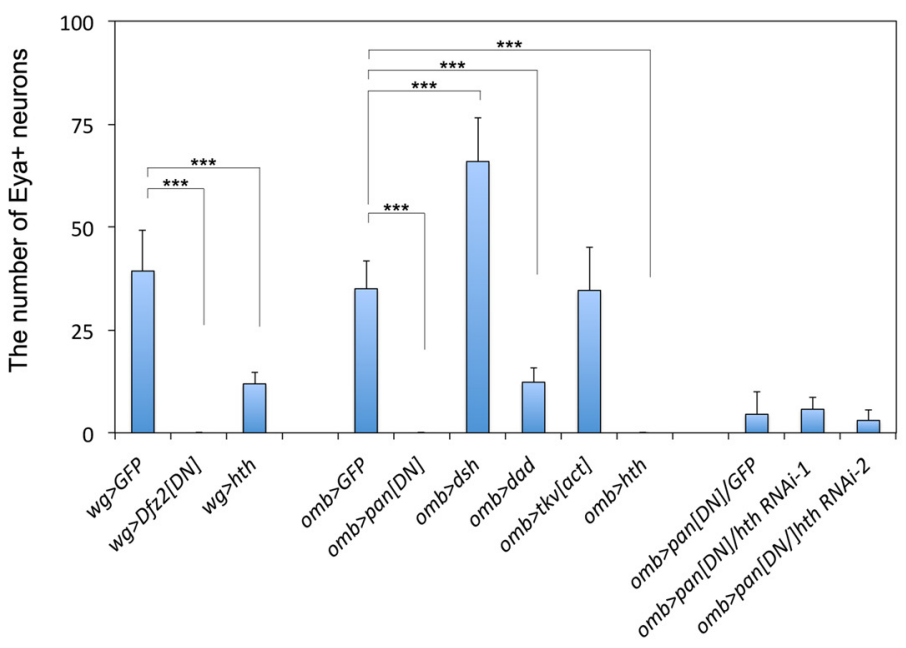

Figure 7. DPP signaling is involved in the regulation of neurogenesis in the GPC region. $\boldsymbol{A}, \mathrm{dpp}$-lacZ (magenta) is expressed in regions adjacent to the GPCs and in Eya ${ }^{+}$( $\left.g r e e n\right)$ neurons as indicated by white arrows. $\boldsymbol{B}$, Expression pattern of Eya (magenta) in omb-Gal4 larvae. C, Expressing Dad under the control of omb-Gal4, the number of Eya ${ }^{+}$(magenta) neurons was significantly reduced (white arrows). D, Expressing constitutive active form of Tkv, the number of Eya ${ }^{+}$(magenta) neurons was not affected. $\boldsymbol{E}$, Quantification of the number of Eya ${ }^{+}$neurons (mean \pm SD; $n=8-15$ ). $h$ th RNAi lines, $h t h^{f F 02733}$ and $h t h^{h M S 01112}$, are designated as $h$ th RNAi-1 and hth RNAi-2, respectively. ${ }^{* * *} p<0.001$ (unpaired two-tailed Student's $t$ test). neurons, we were able to distinguish between GPCs and IPC by the expression of Fas3, an IPC-specific marker. Fas3 is expressed in the IPC but not in the GPC region (Fig. $6 B$, white arrows, indicating no Fas3 expression in the GPCs); thus, the GPCs are Robo $2^{+} / \mathrm{Fas}^{-}{ }^{-}$(Fig. $6 \mathrm{C}$, green), whereas IPC is Robo ${ }^{+} / \mathrm{Fas}^{+}$(Fig. 6C, orange). By using these two markers, we examined the effect of Wnt signaling on GPC formation. First, we suppressed Wnt signaling in the GPCs under the control of wg-Gal4 and found that Robo2 expression (visualized with anti-HA antibody) was lost, whereas ectopic Drf ${ }^{+}$neurons were observed (Fig. $6 D$, white arrows; $n=21$ / 30). Second, when Axin mutant clones were created in the anterior region of the medulla primordium, Robo2 expression was ectopically induced within the clones (Fig. 6E,F; Fig. 6E, white arrows; $n=57 /$ 150). Fas3 expression was not found in almost all Axin mutant clones (Fig. $6 F$; $n=106 / 109)$. These results suggest that ectopic GPCs were induced by the activation of Wnt signaling in the anterior region. Because the ectopic Robo $2^{+}$cells in the Axin mutant clones expressed the NB marker, Dpn, it is likely that ectopic GPCNBs were induced in the region of OPC (Fig. 6E, yellow arrows; $n=7 / 49$ ).

In contrast to the above observations, ectopic Robo2 expression was not found in $h$ th mutant clones in the OPC (Fig. $6 G ; n=54 / 54$ ), suggesting that the loss of Hth is not sufficient to induce the GPC identity.

Another signaling pathway may regulate the cell proliferation in the GPC-NBs to produce Eya ${ }^{+}$neurons. One such candidate is Decapentaplegic (Dpp), a homolog of mammalian BMP-2 or BMP-4 (Wozney et al., 1988), because $d p p-l a c Z$ is specifically expressed in the posterior region of the medulla primordium flanking the GPC region ( $w g$-Gal4 expressing domain, Fig. 7A) (Kaphingst and Kunes, 1994). The number of Eya ${ }^{+}$neurons was significantly decreased by the suppression of Dpp signaling via the induction of Daughters against dpp (Dad), which inhibits Dpp signaling, under the control of omb-Gal4 (Fig. 7C,E; Fig. 7C, white arrows, $n=14 / 19$ ). However, overactivation of Dpp signaling by the induction of a constitutively active form of Thickvein (Tkv), a Dpp receptor, induced no significant increase in $\mathrm{Eya}^{+}$neurons (Fig. $7 D, E$ ). These results suggest that activation of Dpp signaling is necessary, but not sufficient, for the production of $\mathrm{Eya}^{+}$neurons. 


\section{Discussion}

The brain contains a wide variety of neurons, and neuronal diversity is indispensable for its complex higher-order functions. Although all neurons are produced from neuronal stem cells, neuronal stem cells located in different regions produce different types of neurons. Therefore, generating diversity of neuronal stem cells is essential for the production of a wide variety of neurons. In the present study, we demonstrated that Wnt signaling plays essential roles in the determination of the posterior identity of stem cell pools in the fly visual center (Fig. 8). The importance of Wnt signaling is also emphasized in the regulation of stem cell identities in the developing thalamus in mammals (Bluske et al., 2012). Our results suggest that the regulatory mechanisms that determine the identities of neural stem cell pools are conserved between insects and mammals.

\section{Molecular mechanisms of Wnt signaling-mediated specification of neural progenitor pool identity} The progenitor pool in the anterior region of the medulla primordium is the $\mathrm{OPC}$, and the posterior region is the GPCs or tips of the OPC (wg-Gal4+ domain). The GPCs produced OPC-type neurons instead of GPC-type when Wnt signaling was suppressed within the GPC region (Figs. 2, 3). This result suggests that the failure of Wnt signal transduction caused anteriorization of the posterior region of the medulla primordium; and consequently, OPC-type neurons were observed in the GPC region. This finding indicates that Wnt signaling prevents the posterior region from obtaining the OPC identity and, instead, confers GPC identity through the suppression of anteriorization. Because the suppression of Wnt signaling in the posterior region resulted in the transformation from the GPCs to the OPC, the medulla primodium might be solely composed of the OPC by default. By the activation of Wnt signaling in the OPC, the cells located in the anterior region assumed a posterior identity (GPC identity). Our results support these notions because the ectopic activation of Wnt signaling induced an ectopic appearance of the GPCs in the anterior region (Fig. 6).

Wnt signaling is involved in the specification of progenitor pools of mammalian thalamus, pTH-R and pTH-C, and prethalamus (Zhou et al., 2004; Bluske et al., 2012). When $\beta$-catenin is conditionally knocked out in the pTH-C, pTH-R- and prethalamus-type progenitor cells are ectopically observed in the pTH-C. In contrast, pTH-C-type progenitor cells are ectopically induced in the pTH-R by the expression of constitutively active $\beta$-catenin (Bluske et al., 2012). The $\beta$-catenin activity might also be regulated under the control of Wnt ligands in developing thalamus. Thus, the roles of Wnt signaling are quite similar in both the Drosophila visual center and the mammalian thalamus.

The present results suggest that Hth acts as the OPC-defining factor that is repressed under the control of Wnt signaling (Fig. 4). Suppression of Wnt signaling resulted in the ectopic induction of Hth in GPCs, and ectopic Hth expression sufficiently transformed the GPCs to the OPC (Fig. $4 A-F$ ). Although these results suggest

\section{wildtype}
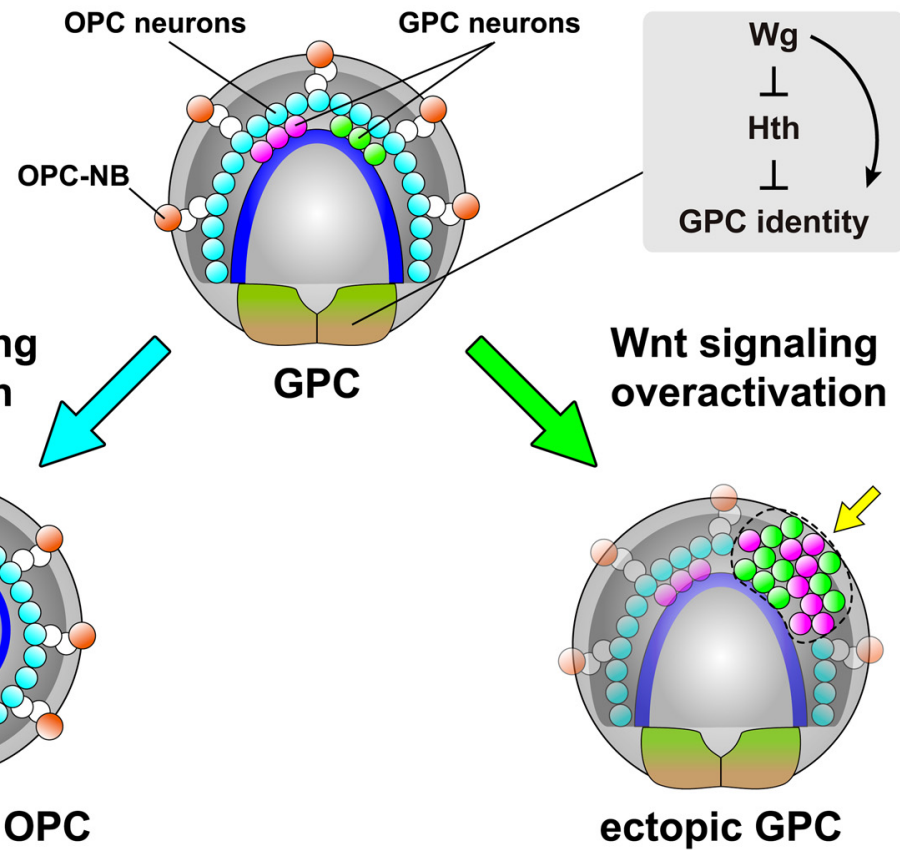

Figure 8. A model of Wnt signaling-mediated specification of neural progenitor pool identity. Schematics of larval medulla. In

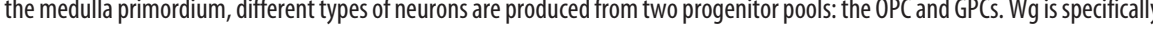
GPC-type neurons (magenta and green). By contrast, OPC obtains GPC identity when Wnt signaling is ectopically activated in anterior region of the medulla (yellow arrow). As a result, GPC-type neurons (green and magenta) are ectopically generated in anterior region instead of $\mathrm{OPC}$-type neurons.

that $\mathrm{Hth}$ is an essential factor for the specification of the OPC identity, Drf ${ }^{+}$neurons were normally observed, and ectopic Eya ${ }^{+}$and Robo ${ }^{+}$cells were absent in $h$ th mutant clones created at the anterior region (Fig. $6 G$ ). Thus, Hth is sufficient, but not necessary, for the specification of the OPC identity. In contrast to the roles of Hth, Wnt signaling is necessary and sufficient for the formation of GPCs and GPC-type neurons because the loss of Eya ${ }^{+}$GPC-type neurons and loss of Robo2 expression caused by the loss of Wnt signaling (Figs. $1 D, 6 D$ ) were only partially rescued by the simultaneous absence of Wnt signaling and Hth expression (Fig. 4J; and data not shown). We cannot exclude the possibility that $h t h$ RNAi did not completely remove $\mathrm{Hth}$ expression and residual $\mathrm{Hth}$ induced the production of Eya ${ }^{+}$neurons. However, it is fairly likely that Wnt signaling induces posteriorization of the medulla directly or through unidentified target genes.

\section{Regulation of neurogenesis in the GPCs}

Wnt signaling is also involved in the regulation of progenitor proliferation in the mammalian cerebral cortex (Chenn and Walsh, 2002) and the ganglionic eminence (Gulacsi and Anderson, 2008). However, this effect may not occur in the medulla primordium because GPC-specific overactivation of Wnt signaling did not result in an increase in Eya ${ }^{+}$neurons. Although an activation of Wnt signaling in the region adjacent to GPCs resulted in a significant increase in $\mathrm{Eya}^{+}$neurons (Fig. $2 E-G$ ), this phenotype was not caused by the increased proliferation of GPC-NBs as visualized by $\mathrm{PH} 3$ expression (data not shown). It is more likely that the GPC region was expanded by the activation of Wnt signaling because omb-Gal4 was expressed in the region adjacent to the GPCs, and consequently, the number of $\mathrm{Eya}^{+}$neurons was increased. 
What regulates the proliferation in the GPC-NBs to produce $\mathrm{Eya}^{+}$neurons? One of the candidates is Dpp (Drosophila homolog of BMP) signaling because suppression of Dpp signaling caused significant reduction in the number of Eya ${ }^{+}$neurons (Fig. 7C). BMP signaling is also involved in the development of the mammalian telencephalon and regulates the differentiation of neurons derived from the ventricular zone ( $\mathrm{Li}$ et al., 1998) and the local interneurons derived from the ganglionic eminence (Mukhopadhyay et al., 2009). Additionally, BMP signaling controls neural stem cell pool identity in the dorsal spinal cord (Jessell, 2000), suggesting that the same signaling pathways are involved in developmental processes in both the Drosophila visual center and the mammalian CNS.

\section{Expanding neural diversity during brain development}

It is crucial to expand neural diversity during brain development. Similarly to neurogenesis in mammals, neuronal types are determined depending on the neuronal birth order, which is under the control of sequential expression of temporal transcription factors in NBs, in both OPC and GPCs (Li et al., 2013; Suzuki et al., 2013; Bertet et al., 2014). If the neurons produced by these two different origins were combined, the neural diversity would be further expanded. Indeed, as observed in mammalian brain development, neuronal populations derived from different origins, OPC and GPCs, orchestrates the formation of neural circuits in the medulla (Bertet et al., 2014; Suzuki et al., 2016a). Therefore, the molecular mechanisms that specify the neural progenitor zone identity between different neuronal origins, such as OPC and GPCs, are very important to understand brain development. The present results suggest that $\mathrm{Wg}$ activates $\mathrm{Wnt}$ signaling and consequently establishes GPC identity in the posterior region of the medulla primordium via the suppression of Hth expression and that Wnt signaling-mediated specification of progenitor pool identity is conserved between insect and mammalian brains (Fig. 8).

\section{References}

Anderson SA, Eisenstat DD, Shi L, Rubenstein JL (1997) Interneuron migration from basal forebrain to neocortex: dependence on Dlx genes. Science 278:474-476. CrossRef Medline

Bertet C, Li X, Erclik T, Cavey M, Wells B, Desplan C (2014) Temporal patterning of neuroblasts controls Notch-mediated cell survival through regulation of Hid or Reaper. Cell 158:1173-1186. CrossRef Medline

Bluske KK, Vue TY, Kawakami Y, Taketo MM, Yoshikawa K, Johnson JE, Nakagawa Y (2012) $\beta$-Catenin signaling specifies progenitor cell identity in parallel with Shh signaling in the developing mammalian thalamus. Development 139:2692-2702. CrossRef Medline

Chenn A, Walsh CA (2002) Regulation of cerebral cortical size by control of cell cycle exit in neural precursors. Science 297:365-369. CrossRef Medline

Colonques J, Ceron J, Tejedor FJ (2007) Segregation of postembryonic neuronal and glial lineages inferred from a mosaic analysis of the Drosophila larval brain. Mech Dev 124:327-340. CrossRef Medline

Fischbach KF, Dittrich APM (1989) The optic lobe of Drosophila melanogaster: I. A Golgi analysis of wild-type structure. Cell Tissue Res 258:441475. CrossRef Medline

Flandin P, Zhao Y, Vogt D, Jeong J, Long J, Potter G, Westphal H, Rubenstein JL (2011) Lhx6 and Lhx8 coordinately induce neuronal expression of Shh that controls the generation of interneuron progenitors. Neuron 70: 939-950. CrossRef Medline

Gulacsi AA, Anderson SA (2008) Beta-catenin-mediated Wnt signaling regulates neurogenesis in the ventral telencephalon. Nat Neurosci 11:13831391. CrossRef Medline

Hasegawa E, Kitada Y, Kaido M, Takayama R, Awasaki T, Tabata T, Sato M (2011) Concentric zones, cell migration and neuronal circuits in the Drosophila visual center. Development 138:983-993. CrossRef Medline

Ito K, Awano W, Suzuki K, Hiromi Y, Yamamoto D (1997) The Drosophila mushroom body is a quadruple structure of clonal units each of which contains a virtually identical set of neurones and glial cells. Development 124:761-771. Medline

Jeong Y, Dolson DK, Waclaw RR, Matise MP, Sussel L, Campbell K, Kaestner KH, Epstein DJ (2011) Spatial and temporal requirements for sonic hedgehog in the regulation of thalamic interneuron identity. Development 138:531-541. CrossRef Medline

Jessell TM (2000) Neuronal specification in the spinal cord: inductive signals and transcriptional codes. Nat Rev Genet 1:20-29. CrossRef Medline Kaphingst K, Kunes S (1994) Pattern formation in the visual centers of the Drosophila brain: wingless acts via decapentaplegic to specify the dorsoventral axis. Cell 78:4437-4448. CrossRef Medline

Kataoka A, Shimogori T (2008) Fgf8 controls regional identity in the developing thalamus. Development 135:2873-2881. CrossRef Medline

Kurant E, Pai CY, Sharf R, Halachmi N, Sun YH, Salzberg A (1998) Dorsotonals/homothorax, the Drosophila homologue of meis1, interacts with extradenticle in patterning of the embryonic PNS. Development 125: 1037-1048. Medline

Li W, Cogswell CA, LoTurco JJ (1998) Neuronal differentiation of precursors in the neocortical ventricular zone is triggered by BMP. J Neurosci 18:8853-8862. Medline

Li X, Erclik T, Bertet C, Chen Z, Voutev R, Venkatesh S, Morante J, Celik A, Desplan C (2013) Temporal patterning of Drosophila medulla neuroblasts controls neural fates. Nature 498:456-462. CrossRef Medline

Mukhopadhyay A, McGuire T, Peng CY, Kessler JA (2009) Differential effects of BMP signaling on parvalbumin and somatostatin interneuron differentiation. Development 136:2633-2642. CrossRef Medline

Rajagopalan S, Vivancos V, Nicolas E, Dickson BJ (2000) Selecting a longitudinal pathway: Robo receptors specify the lateral position of axons in the Drosophila CNS. Cell 103:1033-1045. CrossRef Medline

Sato M, Suzuki T, Nakai Y (2013) Waves of differentiation in the fly visual system. Dev Biol 380:1-11. CrossRef Medline

Soustelle L, Giangrande A (2007) Novel gcm-dependent lineages in the postembryonic nervous system of Drosophila melanogaster. Dev Dyn 236: 2101-2108. CrossRef Medline

Spitzweck B, Brankatschk M, Dickson BJ (2010) Distinct protein domains and expression patterns confer divergent axon guidance functions for Drosophila Robo receptors. Cell 140:409-420. CrossRef Medline

Suzuki T, Sato M (2014) Neurogenesis and neuronal circuit formation in the Drosophila visual center. Dev Growth Differ 56:491-498. CrossRef Medline

Suzuki T, Kaido M, Takayama R, Sato M (2013) A temporal mechanism that produces neuronal diversity in the Drosophila visual center. Dev Biol 380:12-24. CrossRef Medline

Suzuki T, Hasegawa E, Nakai Y, Kaido M, Takayama R, Sato M (2016a) Formation of neuronal circuits by interactions between neuronal populations derived from different origins in the Drosophila visual center. Cell Rep 15:499-509. CrossRef Medline

Suzuki T, Takayama R, Sato M (2016b) eyeless/Pax6 controls the production of glial cells in the visual center of Drosophila melanogaster. Dev Biol 409:343-353. CrossRef Medline

Tuthill JC, Nern A, Holtz SL, Rubin GM, Reiser MB (2013) Contributions of the 12 neuron classes in the fly lamina to motion vision. Neuron 79: 128-140. CrossRef Medline

Vue TY, Aaker J, Taniguchi A, Kazemzadeh C, Skidmore JM, Martin DM, Martin JF, Treier M, Nakagawa Y (2007) Characterization of progenitor domains in the developing mouse thalamus. J Comp Neurol 505:73-91. CrossRef Medline

Vue TY, Bluske K, Alishahi A, Yang LL, Koyano-Nakagawa N, Novitch B, Nakagawa Y (2009) Sonic hedgehog signaling controls thalamic progenitor identity and nuclei specification in mice. J Neurosci 29: 4484-4497. CrossRef Medline

Wozney JM, Rosen V, Celeste AJ, Mitsock LM, Whitters MJ, Kriz RW, Hewick RM, Wang EA (1988) Novel regulators of bone formation: molecular clones and activities. Science 242:1528-1534. CrossRef Medline

Xu Q, Cobos I, De La Cruz E, Rubenstein JL, Anderson SA (2004) Origins of cortical interneuron subtypes. J Neurosci 24:2612-2622. CrossRef Medline

Xu Q, Guo L, Moore H, Waclaw RR, Campbell K, Anderson SA (2010) Sonic hedgehog signaling confers ventral telencephalic progenitors with distinct cortical interneuron fates. Neuron 65:328-340. CrossRef Medline

Zhou CJ, Pinson KI, Pleasure SJ (2004) Severe defects in dorsal thalamic development in low-density lipoprotein receptor-related protein- $6 \mathrm{mu}-$ tants. J Neurosci 24:7632-7639. CrossRef Medline 